\title{
Voluntary Male Circumcision for HIV Prevention in Rural Non-Circumcising Provinces of Northern, Muchinga and Luapula, 2018-2020. A Cross Sectional Survey
}

\section{Ben Chirwa}

Right to Care Zambia

George Magwende

Right to Care Zambia

Philip Mwala

Right to Care Zambia

Ebedy Sadoki

Right to Care Zambia

Frank Chirowa

Right to Care Zambia

Constance Wose Kinge ( $\nabla$ constance.wosekinge@righttocare.org )

Right to Care

Thapelo Maotoe

Right to Care

Charles Chasela

Right to Care

\section{Eula Mothibi}

Right to Care

lan M Sanne

Right to Care

\section{Research Article}

Keywords: Male circumcision, Prevention, HIV testing

Posted Date: September 17th, 2021

DOl: https://doi.org/10.21203/rs.3.rs-743187/v1

License: (c) (1) This work is licensed under a Creative Commons Attribution 4.0 International License. Read Full License 
Page $2 / 13$ 


\section{Abstract}

\section{Introduction}

We describe the VMMC uptake across in Rural Non-circumcising provinces of Luapula, Northern and Muchinga 2018 to 2020.

\section{Method}

This was cross sectional analysis of routine programme data targeting males 10 years older (from October 2018 to April 2020), 15 years (from May 2020) and above VMMC was undertaken using both static and outreach activities. A multi-pronged strategy was adopted that included procurement of VMMC kits to supplement the government's shortfall, training of VMMC providers (doctors, clinical officers and nurses) dedicated space for circumcision in each facility, logistics support ( fuel and allowances) for outreach programs, mapping of catchment areas for community mobilization and demand creation. All circumcisions performed were recorded in $\mathrm{MOH}$ VMMC registers. Data collected was verified by Provincial Health Office. Daily Situation Room (DSR) reports were used to monitor performance. Poor performing districts were followed up and challenges addressed to improve performance.

\section{Results}

A total of 2,130 focused outreach activities were carried out between 2018 and 2020 across the three provinces and 486,750 participants were reached. Of the 486,750 participants reached, 151, 428 were circumcised; 56136 (37\%) from Northern, 49297 (33\%) from Muchinga, and 45995 (30\%) from Luapula. There was a three-fold increase in circumcision between 2018 and 2019 (14,746 circumcisions in 2018 vs 54,287 circumcisions in 2019) and a 14\% increase from 2019 (38\%) to 2020 (52\%; 58,287 circumcisions). Most $(76 \%)$ of the circumcision were done in the age group 15 to 29 years. HIV testing was undertaken among 6,319 participants giving a positivity rate of $2.4 \%$ (149). Improved results were associated with logistical support such as transport, VMMC commodity supplies, and increased dedicated VMMC providers.

\section{Conclusion}

Health systems support including training, logistical support, dedicated space and supply of commodities assisted in increase of VMMC uptake in these rural non-traditional circumcision provinces. even amidst the outbreak of COVID19. Health systems strengthening and community outreach programmes are recommended for building health programs resilience in era of the COVID pandemic.

\section{Introduction}

Voluntary Medical Male Circumcision (VMMC) remains one of the interventions for HIV prevention in countries with high HIV prevalence. Medical Male Circumcision (MMC) is associated with a $60 \%$ reduction in the risk of female-to-male HIV transmission (1-3) although its exact role in HIV transmission 
reduction remains undetermined (4). Male circumcision only offers partial protection against HIV transmission, hence, MMC must be integrated with a comprehensive HIV prevention strategy, which includes treatment for sexually transmitted infections, HIV testing and counselling and promotion of safe sex practices (6) and provision of pre-exposure prophylaxis (PrEP).

Zambia has one of the highest HIV prevalence of $11.1 \%$, women $(14.2 \%)$ remain disproportionately affected than men (7.5\%). Urban areas have higher prevalence, Copperbelt province (15,4\%), Lusaka province $(15.1 \%)$, Central province $(12.4 \%)$ than rural areas, North-Western province $6.1 \%$ Luapula province (7.9\%), Northern province (5.6) and Muchinga province (5.4\%). The National AIDS Strategic Framework 2017-2021 priorities combination HIV prevention with a view to reducing new HIV infections. Zambia introduced VMMC in 2007 with the aim of performing two million circumcisions by the 2020 . The aim was to achieve $80 \%$ coverage of uncircumcised men between ages of 15 and 49 years. Progress to the target was slow and the number of VMMC performed each year fell short of annual targets. The country operational plan for scaleup of VMMC cited 2011 where 84,604 VMMCs represented only $56 \%$ of the 150,000-annual target. Rural provinces such as Northern, Muchinga and Eastern provinces were highlighted to have higher targets but with low circumcision prevalence rates. They were also identified as nontraditional circumcision areas that required greater demand-side efforts to address cultural acceptability, as compared to others. Western and North Western Provinces have smaller population size and cultures that are already more accepting of circumcision. Traditional male circumcision is common among the Lunda and Luvale of North-Western province in Zambia. Traditional circumcision is also viewed as a rite of passage into manhood. Like in South Africa, where traditional circumcision is a common practice among the Xhosa, Sotho, Pedi, Venda and Ndebele cultural groups of South Africa ( 7 , 8). The same practice is common among the tribes of Lunda and Luvale of North Western province in Zambia. This "traditional rite" is usually performed in the months of June, July, November and December, commonly referred to as the circumcision season (4). Traditional circumcision has been associated with surgical complications, increased risk of injury and even death among young men and boys (8).

Right to Care Zambia (RTCZ) though USAID EQUIP and in collaboration with the Zambian Ministry of Health (MoH) is implementing Direct Service Delivery (DSD) since 2018 that included VMMC program across the provinces of Luapula, Muchinga and Northern. In this paper, we describe the uptake and participants characteristics of VMMC across the three provinces from 2018 to 2020.

\section{Materials And Method}

\section{Study Design and study setting}

This was retrospective analysis of routine programme data collected 1st October 2018 to 30th September 2020 and captured into an in-house SmartCare database. The programme setting was in the rural provinces of Northern, Muchinga and Luapula in Zambia, where HIV prevalence is high and male circumcision rate is low. The VMMC program operated in 17 sites across the districts of Chinsali, Mpika, Isoka and Nakonde in Muchinga; Kasama, Luwingu, Mporokoso Kaputa, Mbala in Northern, and Mansa, 
Kawambwa, Samfya Kashikishi and Chembe in Luapula, provinces. The three provinces cater mainly for the rural population with some level of urbanization in the provincial capitals of Mansa, Kasama and Chinsali. There is high population mobility in the border towns of Nakonde at the border with Tanzania, Mpulungu, Kaputa and Mbala in Northern province and Chembe and Nchelenge in Luapula province with Democratic Republic of Congo (DRC). Selection of sites was determined by the funder (USAIDS) and the Ministry of Health at national and provincial levels.

\section{Main Outcome Measures}

The number of circumcisions performed by province over the three-year period and disaggregated by age group, and results of HIV test offered.

\section{Participants}

From October 2018 eligibility included males aged 10 years and above, and through PEPFAR's guidance, from May 2020 the eligibility criteria were revised to 15 years and above. Written consent was required and given independently by all males aged 18 years and above. Boys 16 years but younger than 18 years provided assent to the circumcision procedure after being given information with the parent or legal guardian giving written informed consent. All boys younger than 16 years but above 10 years required a written informed consent of parents or legal guardian to undergo circumcision. The client gave assent, and the parents were required to be present on the day of the circumcision.

\section{Recruitment of participants}

Demand creation activities were undertaken through campaigns at community level, messaging through social media, radio and other forms of media. All those willing were booked and referred to a facility for further screening, consenting and circumcision. At the facility, clients were recruited through walk-ins and referrals from other service points.

\section{Our Approach}

To address the low MMC prevalence rates in these provinces, a multi-pronged strategy was adopted by RTCZ/EQUIP. First, kits to supplement the government's shortfall were procured. Secondly, RTCZ/EQUIP staff were trained to perform circumcisions under the auspices of the $\mathrm{MOH}$ staff. Thirdly, dedicated spaces for circumcision in each facility were identified. All these made it possible to undertake both static and outreach activities concurrently. Districts were supported with logistics such as fuel and allowances for outreach programs. Daily Situation Room (DRC) was used to monitor performance. Poor performing districts were followed up and challenges addressed to improve performance. RTCZ/EQUIP projects staff worked with Ministry of Health VMMC providers to routinely monitor progress of VMMCs performed in the 17 priority facilities. All circumcisions performed were recorded in $\mathrm{MOH} V M M C$ registers. The Ministry of Health and RTCZ/EQUIP used a screening tool to exclude low risk client from HIV testing. All data collected was verified by the Provincial Health Office.

\section{Circumcision procedures}


Once a client was recruited at the facility, the client was registered and provided with general group education. Following group education, individual counselling and HIV testing was offered, and subsequently MMC to all eligible clients Fig. 1 shows an overview of the patient flow.

Pre-operative and circumcision procedures were carried out among clients who accepted and consented. VMMC was deferred for new HIV positive clients with viral load results $>1000$ copies $/ \mathrm{ml}$ in the last six months. For known HIV positive clients, an assessment of the client's adherence to ART and retention in HIV care was conducted. The client was referred to care if there was interruption in treatment (Lost to Follow-up). Eligibility for circumcision for such clients was based on recent VL results not more than six months old. Circumcision was deferred if VL > 1000 copies/ml. The Ministry of Health and RTCZ/EQUIP adopted a screening tool before subjecting clients to circumcision. Those that did not have risk factors such as recent unprotected sex, multiple sex partners were excluded from HIV testing. Hence, the number of circumcisions were not equivalent to the HIV tests done.

\section{VMMC Site team Composition}

Circumcisions were in initially done by surgeons. However, Ministry of Health developed a standard training programme to facilitate delegation of this activity to lower health cadres such as Clinical Officers and Registered Nurses. Thus, the team comprised of a Surgeon (Medical Officer or Clinical Officer), Registered Nurse (RN), two enrolled nurses, a Professional Counsellor, data clerk, a cleaner and a driver on outreach activities.

Post-operative follows up.

Following MMC procedure, follow-up visits were scheduled to assess for any signs of adverse events. The first assessment occurred immediately after the procedure to check for signs of bleeding or any event and if there was no problem, the client was discharged from the facility. The subsequent follow-up visits were scheduled on 2-, 7- and 42-days post-circumcision. Follow up visits were done both in the field and at the clinic.

\section{Data Management and Analysis}

Client data captured by RTCZ data capturers into SmartCare for the period of 1 October 2018 to 30 September 2020 was retrieved and exported into Microsoft Excel. All client identification (name and surname, date of birth, and ID or passport number as well as phone numbers) were deleted. The data was reported in DATIM. Clients younger than 10 years were excluded from the analysis where necessary. Proportions were used to describe the client characteristics as well MMC rates using the de-identified data.

\section{Ethical Consideration}

\section{Informed consent}


or assent was obtained from either the patients or the guardians of the adolescents as part of the programme. Ethics clearance was obtained from the Zambia National Health Research Authority with Ref No: NHRA00001/03/09/2021. All methods were carried out following the World Medical Association Declaration of Helsinki.

\section{Results}

A total of 486,750 clients were reached through outreach activities. Of these, 151,428 consented and were circumcised Most $(n=113,845 ; 75 \%)$ were between ages 15 and 29 years. Of the three provinces, 56136 (37\%) circumcisions were performed in Northern, 49297 (33\%) in Muchinga, and 45995 (30\%) in Luapula. By year, 14,746, 58,787 and 78,395 procedures were done in 2018, 2019, and 2020, respectively (Table 1). There was a threefold increase in circumcision between $2018(14,746)$ and 2019 (58287) and a 14\% increase from 2019 (58287) to 2020 (78395). 
Table 1

Description of participants characteristics

\begin{tabular}{|c|c|c|}
\hline Characteristics & N 157,251 & $\%$ \\
\hline \multicolumn{3}{|l|}{ Age Categories (\%) } \\
\hline $10-14^{\star}$ & 15756 & 10 \\
\hline $15-19$ & 54630 & 36 \\
\hline $20-24$ & 38209 & 25 \\
\hline $25-29$ & 21006 & 14 \\
\hline $30-34$ & 10868 & 7 \\
\hline $35-39$ & 5826 & 4 \\
\hline 40 and Older & 5130 & 3 \\
\hline \multicolumn{3}{|l|}{ Province (\%) } \\
\hline Muchinga & 49297 & 33 \\
\hline Northern & 56136 & 37 \\
\hline Luapula & 45995 & 30 \\
\hline \multicolumn{3}{|l|}{ Year (\%) } \\
\hline 2018 & 14746 & 10 \\
\hline 2019 & 58287 & 38 \\
\hline 2020 & 78395 & 52 \\
\hline \multicolumn{3}{|l|}{ HIV testing offered (\%) } \\
\hline Tested & 6319 & 54.7 \\
\hline Not tested (Known status) & 5224 & 45.3 \\
\hline \multicolumn{3}{|l|}{ HIV Status (\%) } \\
\hline Negative & 6170 & 97.6 \\
\hline Positive & 149 & 2.4 \\
\hline
\end{tabular}

\section{HIV testing}

A total of 6,319 were tested, most were negative $(6,170)$ with $149(2.4 \%)$ tested positive and 5224 had a known status (Table 1). Out of those who tested positive, the majority were among those aged 30-34 
years followed by 25-29 years (Table 2).

Table 2

HIV Status by age $\mathrm{N}=6319$

\begin{tabular}{|lllll|}
\hline Age & Negative N \% & Positive N \% & Known Negative N \% & Total \\
\hline $10-14$ & $1624(89.8)$ & $6(0.3)$ & $178(9.8)$ & 1808 \\
\hline $15-19$ & $2061(88.9)$ & $68(2,9)$ & $190(8.1)$ & 2319 \\
\hline $20-24$ & $1256(89.4)$ & $31(2.2)$ & $118(8.4)$ & 1405 \\
\hline $25-29$ & $676(88.4)$ & $23(3)$ & $66(8.6)$ & 765 \\
\hline $30-34$ & $314(87.0)$ & $16(4.4)$ & $31(8,6)$ & 361 \\
\hline $35-39$ & $128(92.1)$ & $2(1.4)$ & $9(6.5)$ & 139 \\
\hline$\geq 40$ & $111(97.4)$ & $3(2.6)$ & 0 & 114 \\
\hline Total \% & $6170(89.28)$ & $149(2.16)$ & $592(8.56)$ & 6319 \\
\hline
\end{tabular}

\section{Adverse Events}

Minor events reported included pain, post operation swelling and bleeding that were resolved prior to discharge. The programme recorded neither moderate nor severe adverse events. One major adverse event from Mbala, Northern province was severe bleeding requiring referral to the University Teaching Hospital in the capital. The client was diagnosed and treated for haemophilia.

\section{Discussion}

In this analysis, we described the characteristics and VMMC uptake in non-traditional non-circumcising rural provinces of Muchinga, Luapula and Northern. Of the three provinces most of the circumcisions were performed in the Northern Province, followed by Muchinga and Luapula. There was $81 \%$ increase in VMMC uptake in 2020 compared to 2018 when interventions were implemented where uptake was low due to space, shortage of supplies and dedicated providers. The increase is attributed to the provision of dedicated space, additional providers regular supplies and outreach for circumcision camps the numbers. A majority of the VMMC promotions eligible attendees were between the ages 15 to 29 years were offered MMC. Of note was the change of eligible age threshold from 10 years in 2018 to 15 years in 2019. The age majority indicated a young population seeking VMMC. This is in line with another review of VMMC program in nine eastern and southern African countries including South Africa where almost $60 \%$ of the participants were between 15 and 19 years (10). In addition, it reached the young adolescent men, who are sexually active and at risk for HIV acquisition. Out of the three provinces, most circumcisions were undertaken in Northern Province with increasing number of circumcisions over time.

The three-fold increase in VMMC attest to turning around a non-circumcising rural northern region of Zambia into acceptance of VMMC. The ZAMH 2019 reported that coverage of VMMC was more in urban 
(29\%) than in rural areas (14.6.\%). Among rural provinces only North-Western province a known traditional circumcising area had the highest VMMC coverage (39.0\%). In contrast Luapula province $16.4 \%$, Northern province $10.3 \%$ and Muchinga province $12.6 \%$.

Initially all those eligible for MMC were screened for HIV. However, in 2019 the MOH and EQUIP introduced and applied a screening tool to identify those at risk of infection. Hence only those meeting the criteria of risky behaviour were tested. The overall prevalence of HIV was about $2.4 \%$. HIV prevalence was high among those above 25 years and positivity increased with age with a positivity rate of about $4.4 \%$ among those newly diagnosed and between the ages 30-34 The $2.4 \%$ was lower compared to general HIV prevalence in Zambia which is $12.2 \%$ (11), however the rate of positivity was high among the newly tested in the ages above 30 years. The low HIV positivity rate amongst young men of less than 25years is evidence for need to focus efforts for VMMC and other HIV prevention efforts amongst these age groups. All minor events following circumcision resolved prior to discharge. However, we noted one major advent event of post operation haemorrhage. The patient required referral from Northern province to the University Teaching hospital in the capital Lusaka. A diagnosis of haemophilia was made, and appropriate treatment given.

The following limitations are noted: Firstly, being routine program data and not for research purposes, other important variables to inform relationships were missed. However, the large data set offered a realworld experience, thus maximizing generalizability, which often is a challenge in small data sets. Secondly, being routine data, there were several processing challenges due to data capturing, which may lead to misclassification, and subsequent bias. However, manual reviews were conducted, data was verified with source documents thereby improving the quality of the data, giving a more representative and generalizable results.

Increasing VMMC in a traditionally non-circumcision population is a challenge; the challenge is particularly daunting in the light of Zambia target to achieve national $80 \%$ circumcision coverage among males 15 to 49 years.

In conclusion, firstly, health systems interventions, acceptance rates for male circumcision can be increased even in populations that do not have a cultural history of performing cicumcisions. Secondly, the low HIV prevalence and high acceptance rate for circumcision among young adolescent men in the study population indicates the need for VMMC as a method for HIV prevention. Thirdly, the

high HIV positivity and prevalence among those aged 25 and 34 years and above requires intensified demand creation efforts. Lastly, VMMC remains one of the major HIV preventive mechanisms and more demand will help to reach HIV epidemic control.

\section{Abbreviations}

DSD: $\quad$ Direct Service Delivery 
DRC: Democratic Republic of Congo

DATIM: Data for Accountability, Transparency and Impact

EQUIP: $\quad$ Extended Quality Improvement Programme

HIV: $\quad$ Human Immune Deficiency Syndrome

MMC: $\quad$ Medical Male Circumcision

$\mathrm{MOH}: \quad$ Ministry of Health

PEPFAR: President's Emergency Plan for AIDS Relief

PrEP: $\quad$ Pre-exposure Prophylaxis

USAID: United States Agency for International Development

RTCZ: $\quad$ Right to Care Zambia

VMMC: Voluntary Medical Male Circumcision

ZAMH: Zambia Ministry of Health

\section{Declarations}

Ethics approval and consent to participate

Ethical approval was obtained from Zambia National Health Research Authority Ref No:

NHRA00001/03/09/2021. Written informed consent or assent was obtained from all participants included in this study and assent from guardians for those under 16 years of age. All procedure methods were carried out in accordance with the Zambia Ministry of Health guidelines and the World Medical Association Declaration of Helsinki.

Consent for publication

There was no content or images that needed consent.

Availability of data and material

Data for this publication can be accessed by requesting it through the institutional data governance unit by email ( Tapiwa.Mandizvidza@righttocare.org).

Competing interests

The authors declare that they have no competing interests. 


\section{Funding}

This work was supported by USAID EQUIP Grant No. AID-OAA-A-1500070 to Right to Care

\section{Author contributions}

BC: conceptualized the idea, facilitated access to SmartCare and DATIM reviewed the results and the manuscript.

CSC: reviewed and re-wrote the manuscript.

FC: $\quad$ statistical analysis consolidation and reviewed the data and results.

CWK: carried out statistical analysis and reviewed the manuscript

CM: designed the analysis plan, reviewed the data and data analysis.

All authors reviewed and approved the manuscript.

\section{Acknowledgments}

We acknowledge the Zambia MoH staff at facilities for their collaboration and support, and the funding support from the USAID

\section{References}

1. Auvert B, Taljaard D, Lagarde E, Sobngwi-Tambekou J, Sitta R, Puren A. Randomized, controlled intervention trial of male circumcision for reduction of HIV infection risk: The ANRS 1265 trial. PLoS Med. 2005;2(11):1112-22.

2. Bailey RC, Moses S, Parker CB, Agot K, Maclean I, Krieger JN, et al. Male circumcision for HIV prevention in young men in Kisumu, Kenya: a randomised controlled trial. Lancet. 2007;369(9562):643-56.

3. Gray RH, Kigozi G, Serwadda D, Makumbi F, Watya S, Nalugoda F, et al. Male circumcision for HIV prevention in men in Rakai, Uganda: a randomised trial. Lancet. 2007;369(9562):657-66.

4. Connolly C, Simbayi LC, Shanmugam R, Nqeketo A. Male circumcision and its relationship to HIV infection in South Africa: Results of a national survey in 2002. South African Med J. 2008;98(10):789-94.

5. Lagarde E, Dirk T, Puren A, Reathe R-T, Bertran A. Acceptability of male circumcision as a tool for preventing HIV infection in a highly infected community in South Africa. AIDS. 2003;17(1):89-95.

6. Coates TJ, Richter L, Caceres C. Behavioural strategies to reduce HIV transmission: how to make them work better. The Lancet. 2008. p. 669-84. 
7. Auvert B, Lissouba P, Taljaard D, Geffen N, Fiamma A, Heywood M. Key facts on male circumcision. S Afr Med J. 2009;99(3):150-1.

8. Weiss HA, Quigley MA, Hayes RJ. Male circumcision and risk of HIV infection in sub-Saharan Africa: a systematic review and meta-analysis. Aids. 2000;14(15):2361-70.

9. UNAIDS. Joint Strategic Action Framework to Accelerate the Scale-Up of Voluntary Medical Male Circumcision for HIV Prevention in Eastern and Southern Africa. 2011.

10. Seretse MD, Cherutich P, Nkhata A, Come J, Anyolo E, Collen Bonnecwe G, et al. Voluntary Medical Male Circumcision - Southern and Eastern Africa, 2010-2012. MMWR Morb Mortal Wkly Rep. Centers for Disease Control \& Prevention (CDC); 2013;62(47):953-7.

11. Shisana O, Rehle T, Simbayi LC, Zuma K, Jooste S, Zungu N, Labadarios D, D O. South African National HIV Prevalence, Incidence and Behaviour Survey, 2012. HSRC Press. 2014;194.

12. Phili R, Abdool-Karim Q, Ngesa O. Low adverse event rates following voluntary medical male circumcision in a high HIV disease burden public sector prevention program in South Africa. $\mathrm{J}$ Int AIDS Soc. 2014;17:19275.

\section{Figures}

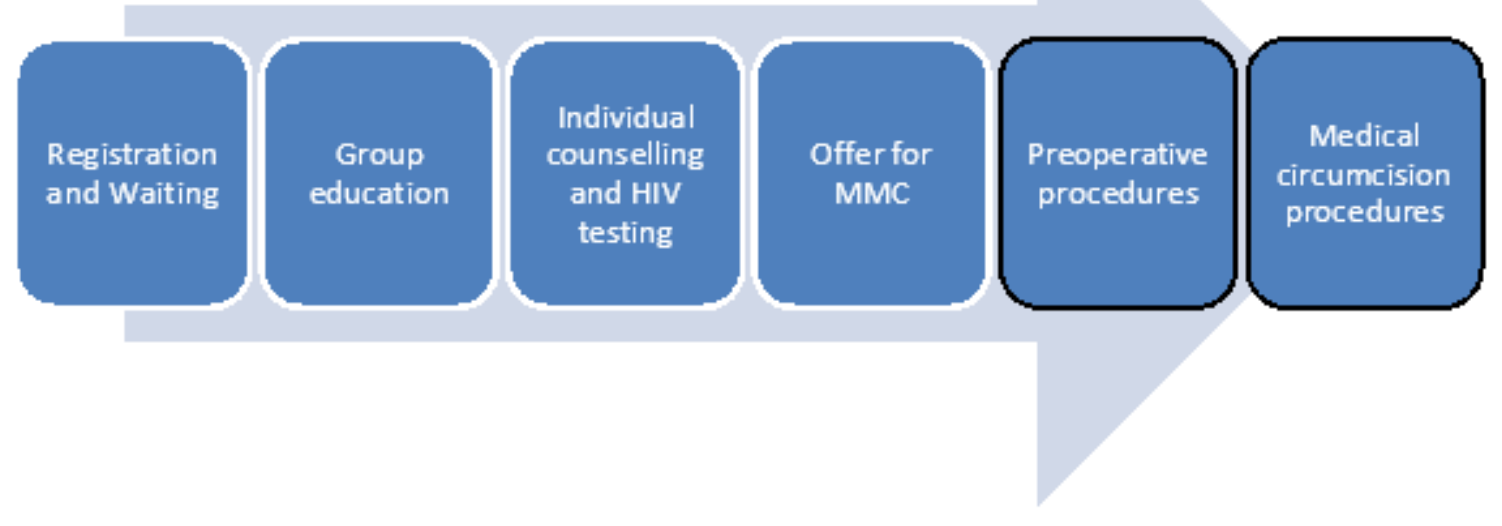

\section{Figure 1}

Flow of activities for MMC at site of operation 